\title{
Antibiotic resistance in invasive Streptococcus pneumoniae isolates identified in Scotland between 1999 and 2007
}

Correspondence

Benjamin Cooke

benjamin.cooke@nhs.net
Received 4 June 2010

Accepted 17 July 2010

\section{Benjamin Cooke, ${ }^{1}$ Andrew Smith, ${ }^{2}$ Mathew Diggle, ${ }^{3}$ Karen Lamb, Christopher Robertson, ${ }^{4}$ Donald Inverarity, ${ }^{5}$ Johanna Jefferies, ${ }^{6}$} Giles Edwards, ${ }^{3}$ Timothy Mitchell, ${ }^{7}$ Stuart Clarke ${ }^{6}$ and Jim McMenamin ${ }^{8}$

\author{
${ }^{1}$ Microbiology Department, Glasgow Royal Infirmary, Glasgow, UK \\ ${ }^{2}$ Infection and Immunity Research Group, Glasgow Dental School, Faculty of Medicine, University of \\ Glasgow, Glasgow, UK \\ ${ }^{3}$ Scottish Haemophilus, Legionella, Meningococcus and Pneumococcus Reference Laboratory, \\ Stobhill Hospital, Glasgow, UK \\ ${ }^{4}$ Department of Statistics and Modelling Science, University of Strathclyde, Glasgow, UK \\ ${ }^{5}$ Department of Microbiology, Monklands Hospital, Airdrie, UK \\ ${ }^{6}$ Molecular Microbiology Group, Division of Infection, Inflammation \& Immunity, University of \\ Southampton, School of Medicine, Southampton, UK \\ ${ }^{7}$ Glasgow Biomedical Research Centre, University of Glasgow, Glasgow, UK \\ ${ }^{8}$ Health Protection Scotland, Glasgow, UK
}

\begin{abstract}
Data from 4727 invasive isolates of Streptococcus pneumoniae submitted to the Scottish Haemophilus, Legionella, Meningococcus and Pneumococcus Reference Laboratory between 1999 and 2007 were analysed to establish susceptibility profiles to penicillin, erythromycin and cefotaxime. Pneumococcal resistance to penicillin over the study period remained low, with only $0.2 \%(n=7 / 4727)$ of isolates falling into this category (MIC $\left.\geqslant 2 \mathrm{mg} \mathrm{I}^{-1}\right)$. These isolates have been sporadic, and have mainly represented serogroup 14 (ST9) and 9 (ST156). In comparison, the 'intermediate sensitivity' group (MIC 0.12-1 $\mathrm{mg} \mathrm{l}^{-1}$ ) ranged between 2 and $6 \%$ per year, the majority from serogroup 9 (ST156). Over the study period, we found that $12 \%(n=585 / 4727)$ of isolates were erythromycin-resistant (MIC $\left.>0.5 \mathrm{mg} \mathrm{l}^{-1}\right)$, with the majority $(n=467 ; 80 \%)$ of these isolates identified as serogroup 14 (ST9). Cephalosporin resistance (cefotaxime MIC $>1 \mathrm{mg} \mathrm{I}^{-1}$ ) was found in only $0.06 \%(n=2 / 3135)$ of isolates. Internationally recognized clones

(Pneumococcal Molecular Epidemiology Network) accounted for $35 \%(n=28 / 81)$ of the penicillin non-susceptible isolates and $75 \%(n=248 / 330)$ of the macrolide-resistant isolates, with ST9 and ST306 predominating. Between 1999 and 2007 we found that $11.6 \%(n=18 / 155)$ of the penicillin non-susceptible isolates and $4.8 \%(n=28 / 585)$ of the macrolide-resistant isolates were from serogroups not covered by the 7-valent conjugate pneumococcal vaccine in use in the UK since 2006. Susceptibility to first-line antimicrobial agents for invasive pneumococcal disease in Scotland remained high over the period 1999-2007.
\end{abstract}

\section{INTRODUCTION}

Penicillin resistance in Streptococcus pneumoniae was first reported in 1967 (Hansman \& Bullen, 1967). Antibiotic resistance is now widespread, and this may contribute to increased morbidity, mortality and cost, although the

Abbreviations: CSF, cerebrospinal fluid; MLST, multilocus sequence typing; PCV-7, 7-valent pneumococcal conjugate vaccine; PMEN, Pneumococcal Molecular Epidemiology Network; ST, sequence type. clinical impact of in vitro resistance varies by disease process. Pneumococcal meningitis caused by penicillinresistant strains responds poorly to penicillin therapy, whereas pneumonia caused by resistant strains may still be successfully treated with penicillin (Song et al., 2004). This is reflected in the differing MIC breakpoints for susceptibility to penicillin for these pathologies (BSAC, 2008).

Rates of reported antimicrobial resistance in the pneumococcus vary greatly, both between and within countries. 
The SENTRY surveillance program demonstrated that less than $20 \%$ of bloodstream infections caused by $S$. pneumoniae were penicillin-resistant (Biedenbach et al., 2004). In comparison, the Alexander Project showed that $57 \%$ of non-invasive isolates from France were penicillin non-susceptible and $50 \%$ were macrolide-resistant (Jacobs et al., 2003). This variability in resistance may be due to a combination of differing patterns of antibiotic use within the community, coverage with the 7-valent pneumococcal conjugate vaccine (PCV-7) (introduced into the childhood vaccination schedule in the UK in September 2006), and spread of internationally recognized antibiotic-resistant clones (McGee et al., 2001). Previous Scottish data from 1988-1999 demonstrated that the prevalence of penicillin intermediate sensitivity among invasive isolates was $2 \%$ and penicillin resistance was $0.04 \%$ (Kyaw et al., 2000).

The aims of this study were to identify the susceptibility profiles to three first-line antibiotics (penicillin, erythromycin, cefotaxime) of $S$. pneumoniae isolates causing invasive pneumococcal disease in Scotland. We establish the contribution that differing serogroups and sequence types (STs) make to the burden of antibiotic resistance and the extent to which these are covered by PCV-7 and PCV13. Finally, we explore the extent to which internationally recognized pneumococcal clones are seen within Scotland.

\section{METHODS}

Isolates. All isolates were obtained from the collection held at the Scottish Haemophilus, Legionella, Meningococcus and Pneumococcus Reference Laboratory, submitted by clinical diagnostic microbiology laboratories from Scotland. Clinical diagnostic microbiology laboratories in Scotland are required to submit all invasive isolates of pneumococci [identified from blood, cerebrospinal fluid (CSF) or other normally sterile sites] for typing and antimicrobial susceptibility testing as part of the ECOSS (Electronic Communication of Surveillance in Scotland) surveillance programme. Isolates without recorded MICs of penicillin and erythromycin were excluded. In total, 4727 isolates were suitable for inclusion, submitted between 1999 and 2007 .

Antimicrobial susceptibility testing. MICs of penicillin, cefotaxime and erythromycin for all isolates were determined using E-test strips (AB Biodisk). Breakpoints published by the British Society of Antimicrobial Chemotherapy were used to assess antimicrobial susceptibility (penicillin resistance $\geqslant 2 \mathrm{mg} \mathrm{l}^{-1}$, intermediate sensitivity $0.12-1 \mathrm{mg} \mathrm{l}^{-1}$; erythromycin resistance $>0.5 \mathrm{mg} \mathrm{l}^{-1}$; cefotaxime resistance $>1 \mathrm{mg} \mathrm{l}^{-1}$ ). The term 'penicillin non-susceptible' refers to all resistant and intermediate-sensitivity isolates.

Typing of isolates. All isolates were serogrouped by co-agglutination, as described by Smart (1986). Full serotyping (a more discriminatory typing method) was carried out on all isolates submitted between 2003 and 2007. Multilocus sequence typing (MLST) (Enright \& Spratt, 1998) was performed using a semiautomated technique on all samples from 2003-2007 $(n=3073)$ (Jefferies et al., 2003). The Pneumococcal Molecular Epidemiology Network (PMEN) strains are international pneumococcal clones which may be associated with increased antibiotic resistance. They were identified by a combination of serotype and MLST (www.sph. emory.edu/PMEN/index.html).

\section{RESULTS AND DISCUSSION}

\section{Penicillin non-susceptibility}

There were 4727 invasive isolates included for analysis, submitted between 1999 and 2007. Pneumococcal resistance to penicillin over the study period remained rare, with only $0.15 \%(n=7,95 \%$ CI $0.07-0.31 \%)$ of isolates falling into this category (MIC $\geqslant 2 \mathrm{mg} \mathrm{l}^{-1}$ ) (Table 1 ). These isolates were sporadic, and were mainly represented by serogroup 14. In comparison, 148 isolates $(3.1 \%, 95 \% \mathrm{CI}$ $2.7-3.7 \%)$ were identified in the 'intermediate sensitivity' group (MIC $0.12-1 \mathrm{mg} \mathrm{l}^{-1}$ ), with an incidence ranging from $6.2 \%$ (95\% CI $3.8-9.9 \%$ ) in 1999 to $2.2 \%$ (95\% CI $1.3-3.5 \%)$ in 2006 , rising to $3.6 \%(95 \%$ CI $2.4-5.4 \%)$ in 2007 (Fig. 1). There is clear evidence of a decreasing trend in penicillin resistance from 1999 to 2003, whereupon the trend was level $(P=0.008)$. Although not a pre-specified hypothesis, there is evidence of a nonlinear trend and a possible increase in 2007 compared to what was expected from the trend between 1999 and $2006(P=0.06)$.

Penicillin non-susceptible organisms accounted for $3 \%$ $(n=155)$ of those analysed during the current 9 year study, which is an increase from the $2 \%$ previously reported in

Table 1. Penicillin and erythromycin resistance among invasive isolates by year $(n=4727$ pneumococci isolated between 1999 and 2007)

\begin{tabular}{|ccccc|}
\hline Year & Total no. of isolates & Intermediate penicillin resistance $[\boldsymbol{n}(\%)]$ & Penicillin-resistant $[\boldsymbol{n}(\%)]$ & Erythromycin-resistant $[\boldsymbol{n}(\%)]$ \\
\hline 1999 & 244 & $15(6.15)$ & $0(0)$ & $41(16.8)$ \\
2000 & 358 & $19(5.31)$ & $0(0)$ & $50(13.97)$ \\
2001 & 515 & $19(3.69)$ & $1(0.19)$ & $83(16.12)$ \\
2002 & 537 & $19(3.54)$ & $1(0.19)$ & $81(15.08)$ \\
2003 & 679 & $15(2.21)$ & $1(0.15)$ & $80(11.78)$ \\
2004 & 517 & $11(2.13)$ & $3(2.13)$ & $65(12.57)$ \\
2005 & 594 & $14(2.36)$ & $0(0)$ & $67(11.28)$ \\
2006 & 697 & $15(2.15)$ & $0(0)$ & $81(11.62)$ \\
2007 & 586 & $21(3.58)$ & $1(0.17)$ & $37(6.314)$ \\
\hline
\end{tabular}


Penicillin non-susceptibility

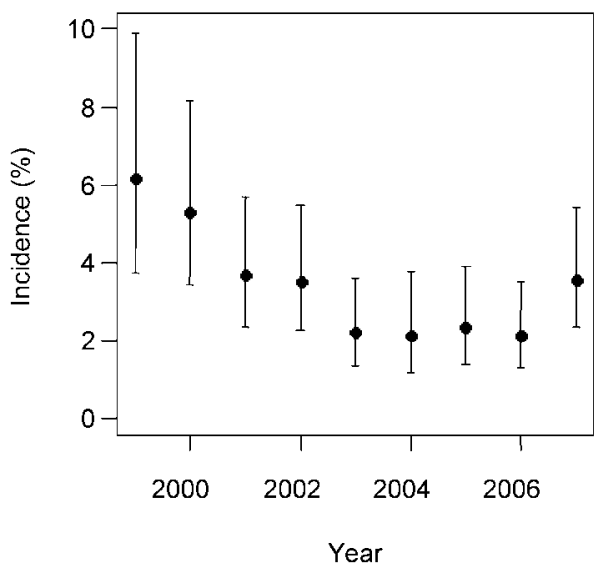

Erythromycin resistance

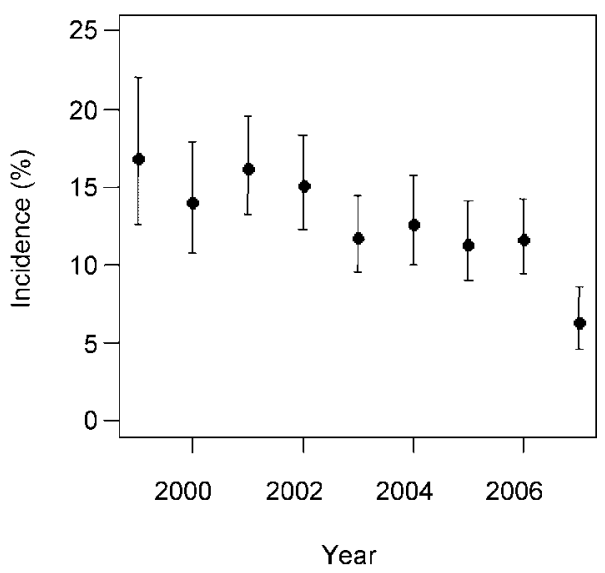

Fig. 1. Penicillin and erythromycin non-susceptibility by year.

Scotland (Kyaw et al., 2000). The 155 penicillin nonsusceptible isolates were isolated mainly from patients over 65 years of age $(n=87,56 \%)$, with 22 samples originating from children under 16 years $(14 \%)$, of which 13 were from children under 2 years of age $(8 \%)$. Non-susceptibility to penicillin varied with age $(P=0.01)$, with nonsusceptibility of $4.1 \%$ (95\% CI $3.3-5.0 \%)$ among those aged 65 and over, $2.4 \%$ (95\% CI $1.8-3.2 \%$ ) among those aged $16-64$, and $3.1 \%$ (95\% CI 2.1-4.7\%) in children.

A small proportion $(n=171 ; 4 \%)$ of the total number of invasive isolates were from CSF, and of these, six (4\%) were of intermediate penicillin sensitivity. In total, 4531 (96\%) of the isolates over the study period were from blood, and 143 of these blood culture isolates (3\%) were non-susceptible to penicillin. There is no evidence that the source of the sample influences sensitivity to penicillin $(P=0.07)$.

The clinical significance of intermediate sensitivity to penicillin in non-meningeal pneumococcal infections is doubtful (Choi \& Lee, 1998), as the levels of drug in the affected tissues are usually higher than the MIC of the organism (Hieber \& Nelson, 1977). Consequently, adequate dosing may still result in good clinical response (Musher et al., 2001). The Clinical and Laboratory Standards Institute breakpoints for pneumococci were revised in 2008 to reflect this feature, and the MIC for non-meningeal pneumococcal resistance is now $\geqslant 8 \mu \mathrm{g} \mathrm{ml}^{-1}$ (CLSI, 2008).

Antibiotic use in primary care in Scotland has increased by $18.3 \%$ between 2004 and 2008 (Health Protection Scotland, 2010). Overall use in 2008 was 19.4 daily defined doses per 1000 of the population per day. Amoxicillin accounted for $24 \%$ of the antibiotics used, with phenoxymethyl penicillin accounting for $4 \%$ and the macrolides (erythromycin and clarithromycin) accounting for $17 \%$ (Health Protection Scotland, 2010). The European Surveillance of Antimicrobial Consumption project found
Scotland to have overall community antibiotic usage in line with the majority of European countries.

Ten serogroups were identified among the 155 nonsusceptible isolates submitted throughout the duration of the study $(1,3,6,8,9,14,19,23,35$ and 38) (Table 2). Of these, five serogroups/types $(1,3,8,35$ and 38$)$ are not covered by the PCV-7 introduced into the UK in 2006. These accounted for $11.6 \%$ of the non-susceptible isolates [ $n=18(95 \%$ CI $7.5-17.6 \%)]$. Forty-seven per cent of all invasive pneumococcal disease cases had serogroups covered by the PCV-7 vaccine. However, seven of those non-susceptible isolates not covered by the vaccine were isolated in 2007 (after the introduction of the PCV).

In the years where full serotype information was available (2003-2007), 11 serotypes were identified from the penicillin non-susceptible isolates $(1,3,4,6 \mathrm{~A}, 6 \mathrm{~B}, 9 \mathrm{~V}, 14$, 19A, 19F, 23F and 35B) (Table 3). The PCV-7 covers 64 of the 77 (typable) non-susceptible strains isolated over this 5 year period $(83 \%)$. This figure rises to $92.2 \%$ with the PCV-13. The principal serotype not covered by the vaccine is $35 \mathrm{~B}(n=6)$.

Five serogroups/types were identified from resistant CSF isolates, of which one (serotype 3 ) is not covered by the conjugate vaccine. Thirty-eight different STs were identified among the 76 penicillin-intermediate isolates from 2003-2007. Of these, ST156 accounted for 18 isolates [23.7\% (95\% CI 15.6-34.4\%)]. The remaining 37 STs were only identified between one and three times.

ST156 accounted for two of the penicillin-resistant clones, and ST246 accounted for one. MLST data were unavailable for the remaining two resistant isolates.

\section{Cephalosporin resistance}

The MICs of cefotaxime were only available for the 3135 isolates submitted between 2003 and 2007. Two resistant 
Table 2. Contribution of different serogroups to penicillin non-susceptible isolates (1999-2007) $(n=4727)$

\begin{tabular}{|lccccc|}
\hline Serogroup & $\begin{array}{c}\text { Intermediate penicillin sensitivity, } \\
\text { from all invasive isolates }[\boldsymbol{n}(\%)]\end{array}$ & $\begin{array}{c}\text { Intermediate penicillin } \\
\text { sensitivity, from CSF }\end{array}$ & $\begin{array}{c}\text { Penicillin-resistant } \\
{[\boldsymbol{n}(\%)]}\end{array}$ & $\begin{array}{c}\text { Covered by } \\
\text { PCV-7 }\end{array}$ & $\begin{array}{c}\text { Covered by } \\
\text { PCV-13 }\end{array}$ \\
\hline 1 & $3(2)$ & 0 & 0 & No & No \\
3 & $1(0.7)$ & 1 & 0 & Yes & Yes \\
4 & 0 & 0 & $1(14.3)$ & Yes & Yes \\
6 & $19(12.8)$ & 1 & $1(14.3)$ & No & Yes \\
8 & $3(2)$ & 0 & $2(28.6)$ & Yes & Yes \\
9 & $50(33.8)$ & 2 & $3(44.9)$ & Yes & Yes \\
14 & $35(23.6)$ & 0 & 0 & Yes \\
19 & $13(8.8)$ & 1 & 0 & No \\
35 & $13(8.8)$ & 1 & 0 & No \\
38 & $6(4.1)$ & 0 & 0 & No \\
Not typed & $1(0.7)$ & 0 & 0 & No \\
\hline
\end{tabular}

NA, Not applicable.

isolates were identified, and both of these were recovered from blood. These were serotype 14 and $22 \mathrm{~F}$, and were isolated from patients aged 1 and 85 years, respectively. Cephalosporin resistance in pneumococci is rare in Scotland ( $0.06 \%$ of isolates). This finding supports current guidelines, which recommend the use of a cephalosporin as first-line therapy in the treatment of bacterial meningitis, with the addition of vancomycin in the case of suspected cephalosporin or penicillin resistance (Chaudhuri et al., 2008).

\section{Macrolide resistance}

Of the 4727 isolates analysed, 585 were erythromycinresistant [12.3\% (95\% CI 11.5-13.3\%)] (Table 1). The majority of these isolates $(95 \%)$ were recovered from blood cultures. There is strong evidence of a reduction in erythromycin resistance since $1999(P<0.0001)$, with $16.8 \%$ (95\% CI 12.6-22.0\%) resistant in 1999 compared to $11.6 \%$ (95\% CI $9.4-14.2 \%)$ in 2006 and $6.3 \%(95 \%$ CI $4.6-8.6 \%$ ) in 2007 (Fig. 1). Although not a prespecified hypothesis, there is evidence of a nonlinear trend and a significant reduction in 2007 compared to what was expected from the reducing trend between 1999 and 2006 $(P=0.005)$.

There were 22 different serogroups isolated, and of these serotype 14 made up the majority [467 isolates, $80 \%$ (95\% CI 76.4-82.9\%)]. The overlap between macrolide and penicillin non-susceptibility was only $0.9 \%(n=44)$. The practice of adding a macrolide to a $\beta$-lactam for the treatment of severe community-acquired pneumonia (Lim et al., 2009) is supported as a means of ensuring that the

Table 3. Contribution of serotypes to penicillin non-susceptible isolates (2003-2007) $(n=3073)$

\begin{tabular}{|lcccc|}
\hline Serotype & $\begin{array}{c}\text { Intermediate penicillin } \\
\text { sensitivity }[\boldsymbol{n}(\%)]\end{array}$ & Penicillin-resistant $[\boldsymbol{n}(\%)]$ & Covered by PCV-7 & Covered by PCV-13 \\
\hline 1 & $1(1.3)$ & 0 & No & No \\
3 & $1(1.3)$ & 0 & Yes & Yes \\
4 & 0 & $1(20)$ & No & Yes \\
$6 \mathrm{~A}$ & $2(2.6)$ & 0 & Yes & Yes \\
$6 \mathrm{~B}$ & $10(13.2)$ & 0 & Yes & Yes \\
$9 \mathrm{~V}$ & $24(31.6)$ & $1(20)$ & Yes & Yes \\
14 & $10(13.2)$ & $3(60)$ & No & Yes \\
$19 \mathrm{~A}$ & $3(3.9)$ & 0 & Yes & Yes \\
$19 \mathrm{~F}$ & $5(6.6)$ & 0 & No & No \\
23F & $10(13.2)$ & 0 & NA & No \\
35B & $6(7.9)$ & 0 & 0 & \\
Not typed & $4(5.3)$ & & \\
\hline
\end{tabular}

NA, Not applicable. 
impact of single-antibiotic resistance is reduced, in addition to providing treatment for 'atypical' organisms. From the 330 erythromycin-resistant isolates between 2003 and 2007, 54 different STs were identified. The most common was ST9, accounting for 225 [68\% (95\% CI $63.0-73.0 \%)$ ] of the erythromycin-resistant isolates.

\section{PMEN clones}

The PMEN strains are international pneumococcal clones identified by a combination of serotype and MLST. This gives more accurate data on clonal spread than serotype alone, as capsular serogroup switching has been described (Jefferies et al., 2004). The PMEN clones may be associated with increased antibiotic resistance (McGee et al., 2001). A previous Scottish study of a selection of 252 pneumococcal isolates submitted to the Scottish Meningococcus and Pneumococcus Reference Laboratory between 1996 and 2003 found only 16 isolates (6\%) of three distinct PMEN clones (Smith et al., 2006). This study found 1124 isolates belonging to PMEN clones from the 3073 isolates submitted between 2003 and 2007 (37\%), and this increase is likely to be due to additions being made to the list of recognized PMEN clones in 2006 (www.sph.emory. edu/PMEN/pmen_clone_collection.html). PMEN clones accounted for 28 of the penicillin non-susceptible isolates identified from 2003-2007 [34.6\%; $n=28 / 81$ (95\% CI $25.1-45.4 \%)]$ and 248 of the macrolide-resistant isolates [75.1\%; $n=248 / 330$ (95\% CI 70.2-79.5\%)] submitted during that time (Table 4$)$. Interestingly, $65 \%(n=53)$ of penicillin non-susceptible isolates and $25 \%(n=82)$ of macrolide-resistant isolates were not identifiable as belonging to currently recognized PMEN clones.

Levels of antimicrobial resistance for pneumococci in Scotland remain relatively low, with a small increase in non-susceptible isolates. Whether this reflects local patterns of antimicrobial prescribing or background fluctuations in serotype and genotype diversity over time remains to be determined (Jefferies et al., 2010). Interestingly, our susceptibility data are influenced by large numbers of isolates reflecting a successful PMEN clone, the England ${ }^{14}$ ST9 strain $(n=239)$. The second most frequent clone isolated, Sweden ${ }^{1}$ ST306 $(n=225)$, was added to the recognized PMEN clone list in 2006, and is the most prevalent serogroup 1 clone in Europe that is not associated with antibiotic resistance (Brueggemann \& Spratt, 2003).

\section{Pneumococcal conjugate vaccine coverage}

Over the study period, $12 \%(n=18 / 155)$ of the penicillin non-susceptible and $9 \%$ of the macrolide-resistant isolates were from serogroups not covered by the PCV-7 (Table 2). The conjugate vaccine was introduced in 2006 in the UK, and so it is not possible from the current data presented here to determine whether there has been serotype replacement among invasive strains by the serotypes not covered by the vaccine, as has been reported in the case of serotype 19A in the USA (Pelton et al., 2007) and Europe (Ardanuy et al., 2009). Only three isolates with serotype 19 A were identified in the current study.

Table 4. PMEN clones isolated between 2003 and 2007 ( $n=3073$ pneumococci isolated in total over the 2003-2007 period)

\begin{tabular}{|c|c|c|c|}
\hline Clone name & No. isolated & Penicillin non-susceptible & Erythromycin-resistant \\
\hline England $^{14}$ ST9 & 239 & 1 & 230 \\
\hline Sweden ${ }^{1}$ ST306 & 225 & 0 & 0 \\
\hline Netherlands ${ }^{8}$ ST53 & 154 & 0 & 1 \\
\hline Netherlands $^{\text {7F }}$ ST191 & 136 & 0 & 0 \\
\hline Netherlands ${ }^{3}$ ST180 & 126 & 1 & 0 \\
\hline Denmark $^{12 \mathrm{~F}}$ ST218 & 81 & 0 & 0 \\
\hline Sweden $^{4}$ ST205 & 51 & 0 & 0 \\
\hline Netherlands ${ }^{18 \mathrm{C}}$ ST113 & 40 & 0 & 0 \\
\hline Netherlands ${ }^{15 B}$ ST199 & 22 & 0 & 0 \\
\hline Spain $^{9 V}$ ST156 & 16 & 14 & 4 \\
\hline $\mathrm{Utah}^{35 \mathrm{~B}}$ ST377 & 10 & 6 & 3 \\
\hline Portugal $^{6 \mathrm{~A}}$ ST327 & 7 & 0 & 0 \\
\hline Netherlands ${ }^{14}$ ST124 & 5 & 1 & 3 \\
\hline Portugal $^{19 F}$ ST177 & 4 & 0 & 0 \\
\hline Poland $^{6 \mathrm{~B}}$ ST315 & 2 & 1 & 2 \\
\hline Spain $^{6 \mathrm{~B}}$ ST90 & 2 & 2 & 2 \\
\hline Spain $^{23 F}$ ST81 & 1 & 1 & 1 \\
\hline Taiwan $^{19 F}$ ST236 & 1 & 1 & 1 \\
\hline Colombia $^{5}$ ST289 & 1 & 0 & 0 \\
\hline Greece $^{6 \mathrm{~B}}$ ST273 & 1 & 0 & 1 \\
\hline Total & 1124 & 28 & 248 \\
\hline
\end{tabular}


In spring 2010, the new PCV-13 was introduced in Scotland (Department of Health, 2010). It additionally covers serotypes $1,3,5,6 \mathrm{~A}, 7 \mathrm{~F}$ and 19A. Only $9 \%(n=14 / 155)$ of penicillin non-susceptible isolates from the current study would not be covered by the PCV-13.

In conclusion, we report relatively low levels of antimicrobial resistance in invasive pneumococci from Scotland to the common first-line antibiotics used in the treatment of pneumococcal infections. The most common serotype demonstrating reduced susceptibility to penicillin was represented by the serogroup 9 and ST156 clones. The most common serotype demonstrating reduced susceptibility to erythromycin was 14 (ST9) (the England ${ }^{14}$ ST9 strain). Whilst there are a large number of PMEN clones represented in our collection of invasive pneumococcal isolates, many of those identified are not associated with resistance to first-line antibiotic agents.

Antibiotic resistance was identified most commonly in serogroups covered by the PCV-7, and non-susceptibility was rare among non-vaccine serogroups. The principal serotype associated with penicillin non-susceptibility not covered by the PCV-7 or the new PCV-13 was 35B. Continued surveillance for emergence of vaccine escape serotypes and their antimicrobial susceptibility is advised and studies investigating the continued low prevalence of antimicrobial-resistant pneumococci in Scotland, whilst the prevalence remains much higher elsewhere in Europe with comparable rates of community antibiotic use, may help shed light on selection pressures for emergence of resistant isolates.

\section{REFERENCES}

Ardanuy, C., Tubau, F., Pallares, R, Calatayud, L., Domínguez, M. A., Rolo, D., Grau, I., Martín, R., Liñares, J. \& other authors (2009). Epidemiology of invasive pneumococcal disease among adult patients in Barcelona before and after pediatric 7-valent pneumococcal conjugate vaccine introduction, 1997-2007. Clin Infect Dis 48, 57-64.

Biedenbach, D. J., Moet, G. J. \& Jones, R. N. (2004). Occurrence and antimicrobial resistance pattern comparisons among bloodstream infection isolates from the SENTRY Antimicrobial Surveillance Program (1997-2002). Diagn Microbiol Infect Dis 50, 59-69.

Brueggemann, A. B. \& Spratt, B. G. (2003). Geographic distribution and clonal diversity of Streptococcus pneumoniae serotype 1 isolates. J Clin Microbiol 41, 4966-4970.

BSAC (2008). BSAC Methods for Antimicrobial Susceptibility Testing, Version 7.1, February 2008. British Society for Antimicrobial Chemotherapy.

Chaudhuri, A., Martin, P. M., Kennedy, P. G. E., Andrew Seaton, R., Portegies, P., Bojar, M. \& Steiner, I. for the EFNS Task Force (2008). EFNS guideline on the management of community-acquired bacterial meningitis: report of an EFNS Task Force on acute bacterial meningitis in older children and adults. Eur J Neurol 15, 649-659.

Choi, E. H. \& Lee, H. J. (1998). Clinical outcome of invasive infections by penicillin-resistant Streptococcus pneumoniae in Korean children. Clin Infect Dis 26, 1346-1354.

CLSI (2008). MIC interpretive standards for Streptococcus pneumoniae. Performance Standards for Antimicrobial Testing, 18th Informational
Supplement, 28, 123. Wayne, PA: Clinical and Laboratory Standards Institute.

Department of Health (2010). Introduction of Prevenar $13^{\mathrm{TM}}$ into the Childhood Immunisation Programme. Letter from Professor David Salisbury, Director of Immunisation, DH, 8 February 2010 (Gateway Reference 13581).

Enright, M. C. \& Spratt, B. G. (1998). A multilocus sequence typing scheme for Streptococcus pneumoniae: identification of clones associated with serious invasive disease. Microbiology 144, 30493060.

Hansman, D. \& Bullen, M. M. (1967). A resistant pneumococcus. Lancet 290, 264-265.

Health Protection Scotland (2010). Scottish Antimicrobial Prescribing Group (SAPG). Report on Antimicrobial Resistance and Use in Humans in 2008. Health Protection Scotland and Information Services Division. http://www.documents.hps.scot.nhs.uk/hai/amr/ annual-report/2008-sapg-amr-report.pdf

Hieber, J. P. \& Nelson, J. D. (1977). A pharmacologic evaluation of penicillin in children with purulent meningitis. N Engl J Med 297, 410-413.

Jacobs, M. R., Felmingham, D., Appelbaum, P. C., Grüneberg, R. N. \& the Alexander Project Group (2003). The Alexander Project 19982000: susceptibility of pathogens isolated from community-acquired respiratory tract infection to commonly used antimicrobial agents. J Antimicrob Chemother 52, 229-246.

Jefferies, J., Clarke, S. C., Diggle, M. A., Smith, A., Dowson, C. \& Mitchell, T. (2003). Automated pneumococcal MLST using liquidhandling robotics and a capillary DNA sequencer. Mol Biotechnol 24, 303-307.

Jefferies, J. M. C., Smith, A., Clarke, S. C., Dowson, C. \& Mitchell, T. J. (2004). Genetic analysis of diverse disease-causing pneumococci indicates high levels of diversity within serotypes and capsule switching. J Clin Microbiol 42, 5681-5688.

Jefferies, J. M., Smith, A. J., Edwards, G. F. S., McMenamin, J., Mitchell, T. J. \& Clarke, S. C. (2010). Temporal analysis of invasive pneumococcal clones from Scotland illustrates fluctuations in diversity of serotype and genotype in the absence of pneumococcal conjugate vaccine. J Clin Microbiol 48, 87-96.

Kyaw, M. H., Clarke, S., Edwards, G. F., Jones, I. G. \& Campbell, H. (2000). Serotypes/groups distribution and antimicrobial resistance of invasive pneumococcal isolates: implications for vaccine strategies. Epidemiol Infect 125, 561-572.

Lim, W. S., Baudouin, S. V., George, R. C., Hill, A. T., Jamieson, C., Le Jeune, I., Macfarlane, J. T., Read, R. C., Roberts, H. J. \& other authors (2009). BTS guidelines for the management of community acquired pneumonia in adults: update 2009. Thorax 64, iiil-iii55.

McGee, L., McDougal, L., Zhou, J., Spratt, B. G., Tenover, F. C., George, R., Hakenbeck, R., Hryniewicz, W., Lefévre, J. C. \& other authors (2001). Nomenclature of major antimicrobial-resistant clones of Streptococcus pneumoniae defined by the pneumococcal molecular epidemiology network. J Clin Microbiol 39, 2565-2571.

Musher, D. M., Bartlett, J. G. \& Doern, G. V. (2001). A fresh look at the definition of susceptibility of Streptococcus pneumoniae to beta-lactam antibiotics. Arch Intern Med 161, 2538-2544.

Pelton, S. I., Huot, H., Finkelstein, J. A., Bishop, C. J., Hsu, K. K., Kellenberg, J., Huang, S. S., Goldstein, R. \& Hanage, W. P. (2007). Emergence of 19A as virulent and multidrug resistant pneumococcus in Massachusetts following universal immunization of infants with pneumococcal conjugate vaccine. Pediatr Infect Dis J 26, 468-472.

Smart, L. E. (1986). Serotyping of Streptococcus pneumoniae strains by coagglutination. J Clin Pathol 39, 328-331. 
Smith, A. J., Jefferies, J., Clarke, S. C., Dowson, C., Edwards, G. F. S. \& Mitchell, T. J. (2006). Distribution of epidemic antibioticresistant pneumococcal clones in Scottish pneumococcal isolates analysed by multilocus sequence typing. Microbiology 152, 361365.
Song, J. H., Jung, S. I., Ki, H. K., Shin, M. H., Ko, K. S., Son, J. S., Chang, H. H., Kim, S. W., Lee, H. \& other authors (2004). Clinical outcomes of pneumococcal pneumonia caused by antibiotic-resistant strains in Asian countries: a study by the Asian Network for Surveillance of Resistant Pathogens. Clin Infect Dis 38, 1570-1578. 